\title{
DESEMPENHO DO HARVESTER NA COLHEITA DE EUCALIPTO EM DIFERENTES ESPAÇAMENTOS E DECLIVIDADES ${ }^{1}$
}

\author{
Elton da Silva Leite², Luciano José Minette³, Haroldo Carlos Fernandes ${ }^{4}$, Amaury Paulo de Souza ${ }^{5}$, \\ Edvaldes José do Amaral ${ }^{6}$ e Elcio das Graça Lacerda ${ }^{7}$
}

\begin{abstract}
RESUMO - Os objetivos deste trabalho foram analisar e quantificar a influência das declividades (baixada - declividade média de $7,76^{\circ}$ e encosta - declividade média de $17,17^{\circ}$ ) e dos espaçamentos de plantio ( $3 \mathrm{~m} \times 2,5 \mathrm{~m}, 3 \mathrm{~m}$ x 3,33 m e $3 \mathrm{~m}$ x 4,0 m) sobre as capacidades produtivas do harvester em povoamentos de eucalipto de primeira rotação. As análises técnica e econômica estão fundamentadas no estudo de tempos e movimentos, nas produtividades e custos de produção do harvester. Os resultados mostraram que a atividade de traçamento consumiu 46\% do ciclo operacional e o rendimento do harvester tendeu a ser maior quando se aumentava o espaçamento e inferior com o incremento da declividade. Em média, o espaçamento 3 m x 2,5 m e 3 m x 3,33 m gerou um custo maior da ordem de $11 \%$ e $2 \%$ a maior em detrimento do espaçamento 3 m x 4 m. Em relação à declividade, o detalhamento desse impacto negativo gerado pela encosta foi de $11 \%$ em relação à baixada.
\end{abstract}

Palavras-chave: Corte florestal; Produtividade; Custos.

\section{PERFORMANCE OF THE HARVESTER ON EUCALYPTUS CROPS IN DIFFERENT SPACING AND SLOPES}

\begin{abstract}
The objectives of this study were to analyze and quantify the influence of slopes (lowland average slope of $7.76^{\circ}$, and hillside average slope of $\left.17.17^{\circ}\right)$ and planting spacings $(3 \mathrm{~m} \times 2.5 \mathrm{~m}, 3 \mathrm{~m} \times 3.33 \mathrm{~m}$ and $3 \mathrm{~m} \times 4.0 \mathrm{~m})$ on the productive capacities of the harvester in eucalyptus plantation in the first rotation. The technical and economic analysis is based on time and motion study, crop yields and production costs of the harvester. The results showed that the activity of tracing consumed $46 \%$ of the operational cycle, and the harvester's productivity tended to be greater when the spacing increased, and lower with increasing slope. On average, the spacing $3 m \times 2.5 m$ and $3 m \times 3.33 m$, generated a higher cost of about $11 \%$ and $2 \%$ higher over the spacing $3 m \times 4 m$. Regarding the slope, the details of this negative impact generated by the hillside was $11 \%$ when compared to the lowlands.
\end{abstract}

Keywords: Forest cutting; Productivity; Costs.

\section{INTRODUÇÃO}

A colheita florestal em áreas acidentadas, ou em condições topográficas desfavoráveis, exige alto nível de planejamento e detalhamento. Também, é necessário o desenvolvimento de máquinas e equipamentos específicos para essas condições, com os objetivos de minimizar os custos, diminuir a necessidade de mão de obra e aumentar a produtividade (LIMA; LEITE, 2008).
No Brasil, as florestas plantadas para o uso industrial ocupam 6,127 milhões de hectares (ABRAFF, 2009), estando como um dos primeiros países do mundo em reflorestamento do gênero Eucalyptus (MINETTE, 1988).

A mecanização planejada no setor florestal contribui significantemente para maximizar retornos em geral, atendendo aos critérios das certificadoras, que são afetados, principalmente, pela etapa de colheita florestal.

\footnotetext{
${ }^{1}$ Recebido em 15.11.2010 aceito para publicação em 07.11.2013.

${ }^{2}$ Universidade Federal do Recôncavo da Bahia, UFRB, Brasil. E- mail:<elton@ufrb.edu.br>.

${ }^{3}$ Departamento de Engenharia Elétrica e de Produção da Universidade Federal de Viçosa, UFV, Brasil. E-mail:<minette@ufv.br>.

${ }^{4}$ Departamento de Engenharia Agrícola da Universidade Federal de Viçosa, UFV, Brasil. E-mail: $<$ haroldo@ufv.br $>$

${ }^{5}$ Departamento de Engenharia Florestal da Universidade Federal de Viçosa, UFV, Brasil. E-mail:<amaury@ufv.br>.

${ }^{6}$ Celulose Nipo Brasileira S. A., CENIBRA, Brasil.

${ }^{7}$ Instituto Federal de Santa Teresa, IFES, Brasil. E-mail:<elciodgl@hotmail.com>
}

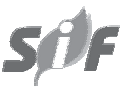

Revista Árvore, Viçosa-MG, v.38, n.1, p.000-000, 2014 
Pulkki (2006) conceituou a operação de corte como a separação da árvore da cepa a partir do seu lugar de crescimento. Segundo Sant'Anna (2008), nessa operação estão compreendidos a derrubada, o desgalhamento, o traçamento e o empilhamento, caracterizando como a etapa que demanda maiores custos financeiros.

Malinovski et al. (2006) identificaram 35 principais variáveis de influência na produtividade das máquinas de colheita de Pinus e 37 principais variáveis de influência na produtividade das máquinas de colheita de eucaliptos, relacionadas ao povoamento, ao terreno e ao planejamento das operações.

Um dos principais aspectos a considerar, no estabelecimento de povoamentos florestais, é a adequação da densidade do plantio, além do arranjo espacial das árvores, de modo a satisfazer as necessidades do produtor florestal e do mercado. Além dessas características, Akay et al. (2004) completaram que os sistemas de colheita devem estar relacionados aos tipos de máquinas, tamanho e volume da árvore e intensidade das operações de colheita.

Martins et al. (2009) concluíram que o volume médio por árvore foi a variável que melhor explicou a capacidade operacional efetiva do harvester. Em seguida, espaçamentos maiores resultam em maior volume individual por árvore, que, consequentemente, influi no melhor desempenho do harvester.

Bramucci e Seixas (2002) e Burla (2008) afirmaram que o aumento na densidade da floresta implica diretamente redução do volume individual das árvores e maior volume por hectare, o que resulta em queda de produtividade do harvester, sendo esse um dos principais fatores que influenciam negativamente a produtividade de equipamentos de colheita florestal (ELIASSON, 1999; GINGRAS, 1992).

Conforme Lima (2008), a declividade do terreno é uma das variáveis operacionais mais importantes a se considerar na mecanização florestal. Segundo Silva et al. (2008), a topografia afeta diretamente o rendimento das máquinas nas operações florestais.

Em relação à declividade, pode-se dizer que, quanto maior a declividade, maiores as limitações ao deslocamento da máquina no terreno e, por conseguinte, menor a produtividade (MALINOVSKI, 2006).
Stampfer e Loschek (1999), estudando a influência do volume da árvore e da inclinação do terreno sobre a produtividade de um harvester de esteiras, concluíram que a produtividade diminui com o aumento da inclinação do terreno.

Objetivou-se com este experimento analisar técnica e economicamente a influência da declividade (baixada e encosta) e do espaçamento do plantio ( $3 \mathrm{~m} \mathrm{x} 2,5 \mathrm{~m}$; $3 \mathrm{~m}$ x 3,33 m; $3 \mathrm{~m}$ x 4,0 m) na colheita de eucalipto com harvester, analisando os ciclos operacionais, rendimentos, custos operacionais e de produção.

\section{MATERIAL E MÉTODOS}

\subsection{Descrição da área de estudo}

A pesquisa foi realizada na região de Belo Oriente, MG, em uma área de 8,43 ha, pertencente à Celulose Nipo Brasileira S. A. - CENIBRA, tendo como referência as coordenadas UTM 766.716 m W e 7.857.186 m S, zona 23 Sul, datum SAD-1969.

O experimento foi conduzido em povoamentos de clones híbridos de eucalipto de alta produtividade em regime de primeira rotação com 7 anos de idade.

Os solos florestais dos talhões são classificados como Cambissolo Latossólico na região de baixada e Neossolo Flúvico na encosta.

\subsection{Descrição e operação do harvester}

O harvester é uma máquina que processa as árvores individualmente, ou seja, cada árvore é derrubada, desgalhada, destopada e traçada separadamente (JACOVINE, 2005).

Utilizou-se o harvester John Deere modelo 1470D, motor John Deere 6090, potência de 241 hp (159,6 $\mathrm{kW}$ ), chassi articulado, transmissão power-shift e cabeçote harvester modelo 270 John Deere, com capacidade de corte de 0,04 m até 0,62 cm de diâmetro, tração $6 \times 6$, com esteiras unindo os pneus frontais em tandem (26.5-20) e traseiros (34-16), proporcionando maior aderência e menor compactação do solo, conforme mostrado na Figura1.

Realizou-se a operação em dois turnos, das 8 às $16 \mathrm{~h}$ e das 16 às $24 \mathrm{~h}$, no período de 2 a 22 de junho de 2009. Avaliou-se a produtividade do harvester no sistema Cut-to-lenght, como proposto pela FAO, sendo o comprimento de tora de $4,40 \mathrm{~m}$, operando em uma faixa 


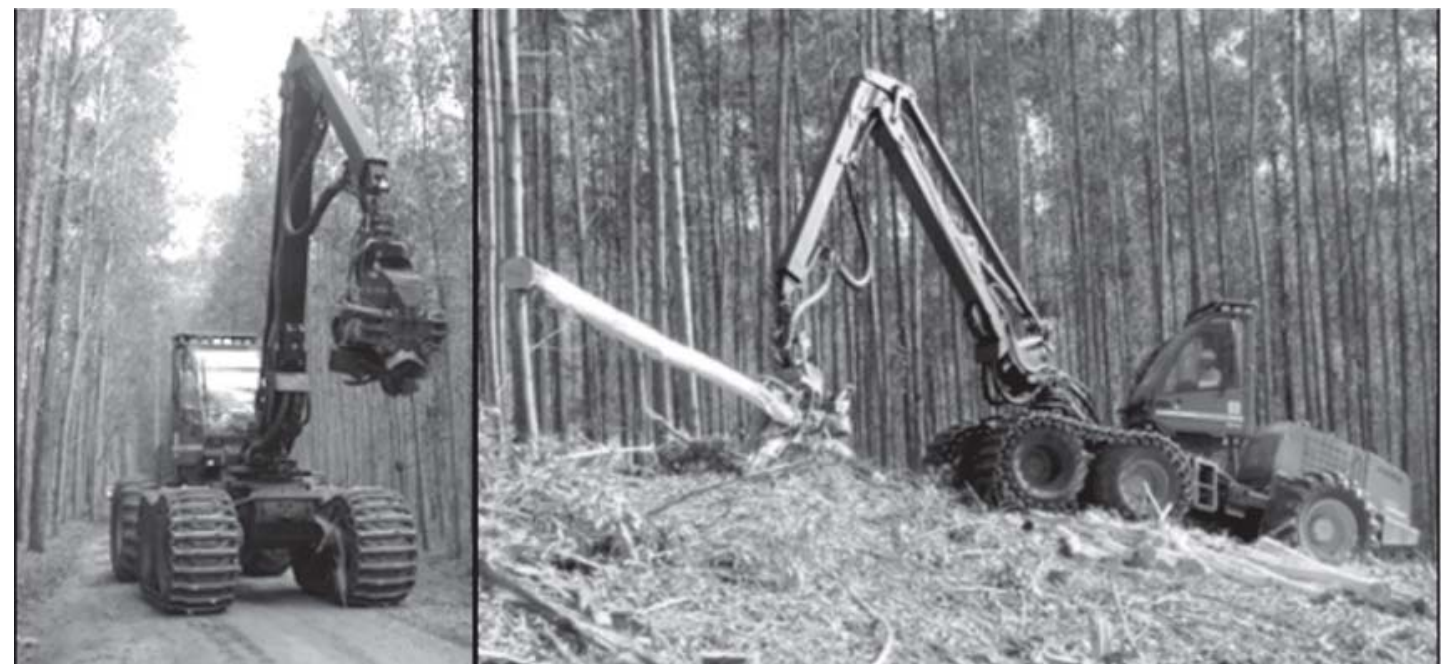

Figura 1 - Vistas frontal e lateral do Harvester John Deere, modelo 1470D. Figure 1 - Front and side views of the John Deere Harvester, model 1470D.

de corte de quatro linhas, sempre se deslocando sobre a segunda linha, onde se depositou a galhada, método mais eficiente segundo Valverde et al. (1996). A derrubada da faixa de corte realizou-se pela segunda linha, seguida da primeira, terceira e quarta linhas.

$\mathrm{O}$ traçamento foi realizado à margem da primeira linha da faixa de corte e os toretes, posicionados em feixes direcionados perpendicularmente à máquina.

\subsection{Características do povoamento}

As unidades amostrais eram compostas por aproximadamente 96 árvores, sendo representadas por oito linhas com 12 árvores em cada, compondo, assim, duas faixas de corte.

Na Tabela 1, mostram-se os dados do inventário, em que as unidades amostrais apresentaram $87 \%$ de árvores sobreviventes. Na transformação do volume de madeira com casca (cc) para volume de madeira sem casca (sc), considerou-se uma redução de 13\% em volume para o clone.

\subsection{Análise de tempos e movimentos}

Para determinação da análise do ciclo operacional do harvester, utilizou-se a marcação pelo método de multimomento. As observações visuais foram efetuadas a cada $15 \mathrm{seg}$, com o auxílio de um cronômetro digital sexagesimal, descrevendo-se a atividade exercida no formulário de dinâmica operacional.
As atividades consideradas na dinâmica deste trabalho foram: deslocamento da máquina - considerouse o deslocamento da máquina na parcela; posicionamento do cabeçote para corte - referiuse ao tempo de direcionamento do cabeçote para iniciar o corte de abate da árvore; corte de derrubada - referiu-se ao acionamento do sabre até o seccionamento do fuste; tombamento da árvore considerou-se o tempo gasto após o corte até que a árvore tocasse o solo ou quando se iniciava o descascamento da árvore; descascamento/ desgalhamento - considerou-se como o tempo decorrido em que os rolos e facas deslizaram sobre o tronco da árvore, descascando-a e desgalhandoa; traçamento - referiu-se ao acionamento do sistema de corte do cabeçote, à medição e ao corte, propriamente dito, até o último torete; manobra - referiu-se aos movimentos da máquina para o retorno do final da parcela para o início dela até o momento em que a máquina se encontrava na frente da próxima faixa de corte da parcela; pausa técnica - considerouse o tempo gasto com ajuste de corrente, comunicação via rádio; pausa pessoal - compreendeu-se o período referente às necessidades fisiológicas dos operadores; limpeza - referiu-se ao deslocamento do cabeçote no local de formação dos feixes, realizando a limpeza do local e depositando o material orgânico na linha de tráfego do harvester.

Revista Árvore, Viçosa-MG, v.38, n.1, p.000-000, 2014 
Tabela 1 - Dados médios de volume por árvore, em metro cúbico com casca ( $\left.\mathrm{m}^{3} \mathrm{cc} / a ́ r v.\right)$ aos 7 anos, e quantidades das parcelas em relação aos blocos e tratamentos experimentais.

Table 1 - Average data of volume per tree, in cubic meters with bark ( $m^{3} \mathrm{cb} /$ tree.) at 7 years age, and quantities of plots in relation to blocks and treatments.

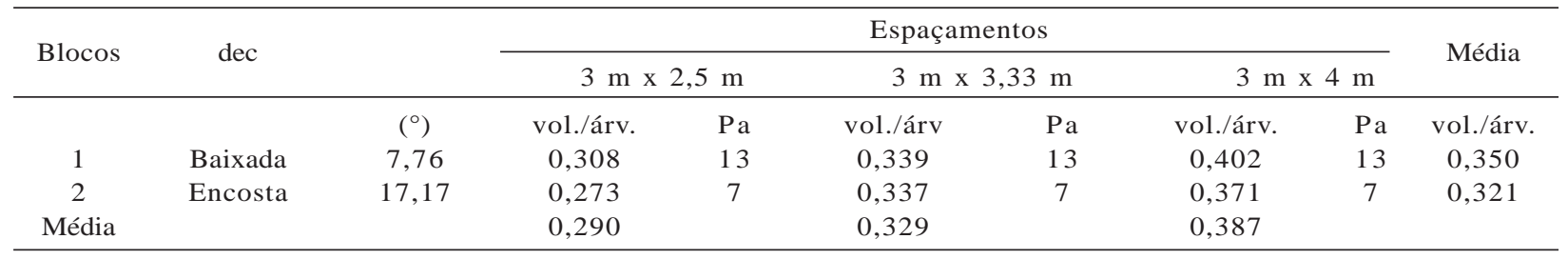

dec = declividade; vol./árv. = volume por árvore $\left(\mathrm{m}^{3}\right)$; e $\mathrm{Pa}=$ número de parcelas. dec $=$ slop; vol./árv. $=$ volume per tree $\left(\mathrm{m}^{3}\right)$; and $\mathrm{Pa}=$ number of plots.

\subsection{Determinação da produtividade, custo operacional e custo de produção}

A produtividade do harvester em $\mathrm{m}^{3} \mathrm{sc} \mathrm{h}^{-1}$ é determinada igualmente ao volume de madeira da parcela sem casca dividido pelas horas efetivamente trabalhadas (SILVA et al., 2010).

Os custos operacionais da máquina foram determinados segundo a metodologia proposta pela FAO, utilizada por Burla (2008). Esses custos foram divididos em custos fixos, custos variáveis e custo de administração, expressos em dólares por hora efetiva de trabalho (US\$ $\mathrm{h}^{-1}$ ) e calculados a partir de análises do banco de dados da empresa, para maior confiabilidade.

O custo de produção da máquina, expresso em US $\$ \mathrm{~m}^{-3} \mathrm{Sc}^{-1}$, foi determinado pela divisão dos custos operacionais (US\$ h) em relação à produtividade $\left(\mathrm{m}^{3} \mathrm{sc} \mathrm{h}^{-1}\right)$.

\subsection{Delineamento estatístico}

O delineamento experimental usado foi o de blocos casualizados, compreendidos em duas faixas de declividade, baixada com declividade média de $7,76^{\circ}$, variância $2,26^{\circ}$ e coeficiente de variação de $16,39 \%$ e a da encosta com declividade média de $17,17^{\circ}$, variância de $1,28^{\circ}$ e coeficiente de variação de $6,58^{\circ}$, determinada usando um clinômetro Eletrônico Digital Hec Haglof Sweden Profissional. Os espaçamentos $3 \mathrm{~m} \times 2,5 \mathrm{~m}$, $3 \mathrm{~m} \times 3,33 \mathrm{~m}$ e $3 \mathrm{~m} \times 4 \mathrm{~m}$ compuseram os três níveis de tratamentos do experimento.

Os resultados do ciclo operacional e da produtividade foram submetidos à análise de variância a 5\% de probabilidade, pelo teste F. Nos casos em que houve diferença estatística significativa, aplicou-se o teste de média de Tukey a 5\% de probabilidade, na análise qualitativa, por meio do software estatística 7.

\section{RESULTADOS}

\subsection{Análise de tempo e movimento}

Os tempos e movimentos estão detalhados em horas por hectare (Tabela 2). Apenas os elementos parciais posicionamento do cabeçote para corte, corte de derrubada, deslocamento, traçamento e limpeza são diferentes estatisticamente pelo teste de Tukey a 5\% de probabilidade.

Evidencia-se que os dados seguem a tendência de declínio à medida que aumenta o espaçamento e reduz a declividade. Pode-se confirmar também que o traçamento tem maior consumo de tempo (47\%), sequenciado pelo posicionamento do cabeçote para corte (17\%) e descascamento/desgalhamento (14\%). Para as atividades de deslocamento da máquina, corte de derrubada e limpeza, toma-se muito pouco tempo durante o processo.

O tempo gasto, em horas, por hectare $\left(\mathrm{h} \mathrm{ha}^{-1}\right)$ é de aproximadamente 10,10; 8,06; e 7,00 na baixada e 11,$48 ; 8,80$; e 7,49 na encosta, para os respectivos espaçamentos de $3 \mathrm{~m}$ x 2,5 m; $3 \mathrm{~m}$ x 3,33 m; e $3 \mathrm{~m} \mathrm{x}$ $4,0 \mathrm{~m}$.

A ordem de corte das árvores na operação frequentou primeiramente a segunda faixa, seguida da primeira, terceira e quarta faixas. Em média, cada avanço do harvester realizava o corte e processamento de 6,07; 5,43; e 5,22 árvores, e o número de árvores por feixe foi de 11,65; 11,09; e 11,22 árvores, nos espaçamentos de $3 \mathrm{~m}$ x 2,5 m; $3 \mathrm{~m} \mathrm{x} \mathrm{3,33} \mathrm{m;} \mathrm{e} 3 \mathrm{~m}$ x 4,0 m. 
Tabela 2 - Análise estatística das médias dos tratamentos, em minutos por hectare (min ha-1), dos elementos parciais que compõem o ciclo operacional do Harvester.

Table 2 - Statistical analysis of treatment means, in minutes per hectare (ha min $^{-1}$ ), the partial elements that make up the operating cycle of the Harvester.

\begin{tabular}{|c|c|c|c|}
\hline \multirow{2}{*}{ ECO(min/ha) } & \multicolumn{3}{|c|}{ Tratamentos } \\
\hline & $3 \mathrm{~m} \times 4 \mathrm{~m}$ & $3 \mathrm{~m} \mathrm{x} \mathrm{3,33} \mathrm{m}$ & $3 \mathrm{~m} \times 2,5 \mathrm{~m}$ \\
\hline $\mathrm{DM}$ & 13,51 a & $17,31 \mathrm{ab}$ & $20,21 b$ \\
\hline $\mathrm{PC}$ & 70,29 a & 87,04 b & 115,12 c \\
\hline Co & 17,41 a & $24,16 b$ & 29,06 с \\
\hline TA & 30,29 a & 35,54 a & 46,03 а \\
\hline Ds & 63,26 a & $71,38 \mathrm{ab}$ & $86,55 \mathrm{~b}$ \\
\hline $\operatorname{Tr}$ & 202,64 a & $237,30 \mathrm{ab}$ & $286,08 \mathrm{~b}$ \\
\hline MM & 10,56 a & 12,11 a & 15,20 a \\
\hline $\mathrm{PT}$ & 0,47 a & 1,45 a & 3,15 a \\
\hline $\mathrm{Lm}$ & 21,28 a & $27,64 \mathrm{~b}$ & $30,08 \mathrm{c}$ \\
\hline
\end{tabular}

As médias da linha seguidas da mesma letra não diferem estatisticamente entre si, pelo teste de Tukey a 5\% de probabilidade. ECO elementos do ciclo operacional; DM - deslocamento da máquina; PC - posicionamento do cabeçote para corte; Co - corte de derrubada; TA - tombamento da árvore; Ds - descascamento/desgalhamento; $\mathrm{Tr}$ - traçamento; MM - manobra da máquina; PT - pausa técnica; and Lm - limpeza.

\subsection{Produtividade}

Na Figura 3, apresentam-se os rendimentos médios do harvester em relação aos blocos e tratamentos. A produtividade na baixada e no espaçamento de 3 x $4 \mathrm{~m}$ é superior, sendo de $31,50 \mathrm{~m}^{3} \mathrm{sc} \mathrm{h}^{-1}$ pelo teste de Tukey a $5 \%$ de probabilidade.

\subsection{Custo operacional}

O custo operacional do harvester foi igual a 145,29 US\$ $\mathrm{h}^{-1}$ em todas as variáveis do experimento. A Figura 2 representa o percentual dos itens que compõem o custo operacional, com uma taxa real de juros de $12 \%$, eficiência operacional de $80 \%$ e cotação de US\$1,00 igual a $\mathrm{R} \$ 1,885$.

Para o cálculo desse custo total, a representatividade dos custos fixos, variáveis e de administração foi, respectivamente, de 24,68 US\$ he ${ }^{-1} ; 113,06$ US $\$$ he $^{-1}$; e 7,57 US $\$$ he$^{-1}$. O custo de administração foi considerado como dados médios da empresa.

\subsection{Custo de produção}

Para os custos de produção de madeira cortada e processada pelo harvester, em comparação com a sua produtividade, observam-se tendência inversa entre os níveis de produtividade e o custo de produção por $\mathrm{m}^{3}$ produzido e diretamente influenciada pelas variáveis do terreno, como a declividade (Figura 3), confirmado por Malinovski et al. (2006), Huyler e Ledoux (1999) e Simões (2010).

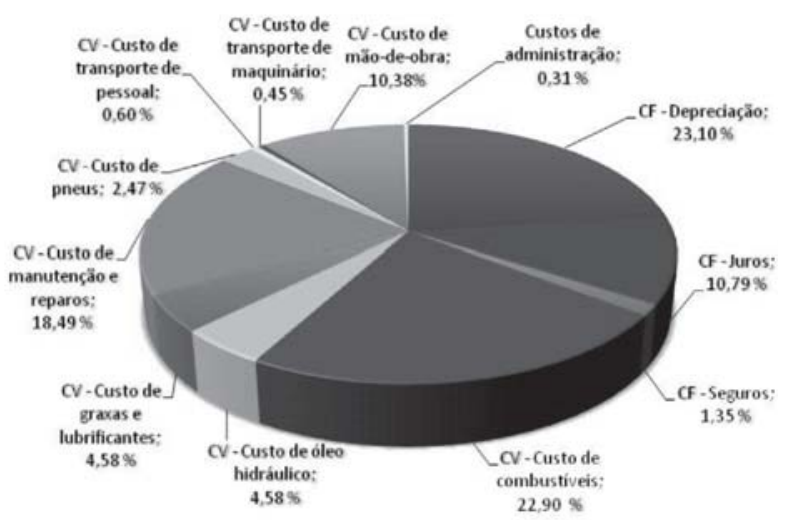

Figura 2 - Distribuição porcentual dos itens que compõem o custo operacional do Harvester. CF = custos fixos; e CV= custos variáveis.

Figure 2-Distribution of items that constitute the operational cost of the Harvester. $C F=$ costs fixos; and $C V$ $=$ costs variables.

Na Figura 3, verifica-se tendência de queda nos custos de produção, à medida que se desloca da encosta para a baixada e quando se aumenta o espaçamento entre árvores, explicado pelo teste de Tukey a 5\% de probabilidade. O módulo é composto pela baixada e pelo espaçamento de $3 \mathrm{~m} \mathrm{x} 4 \mathrm{~m}$ e possui o menor custo: US\$ 4,81 $\mathrm{m}^{-3} \mathrm{SC}$.

\section{DISCUSSÃO}

Fazendo comparação, Burla (2008) obteve rendimento médio de $28,50 \mathrm{~m}^{3} \mathrm{~h}^{-1}$. Já Simões (2010) 


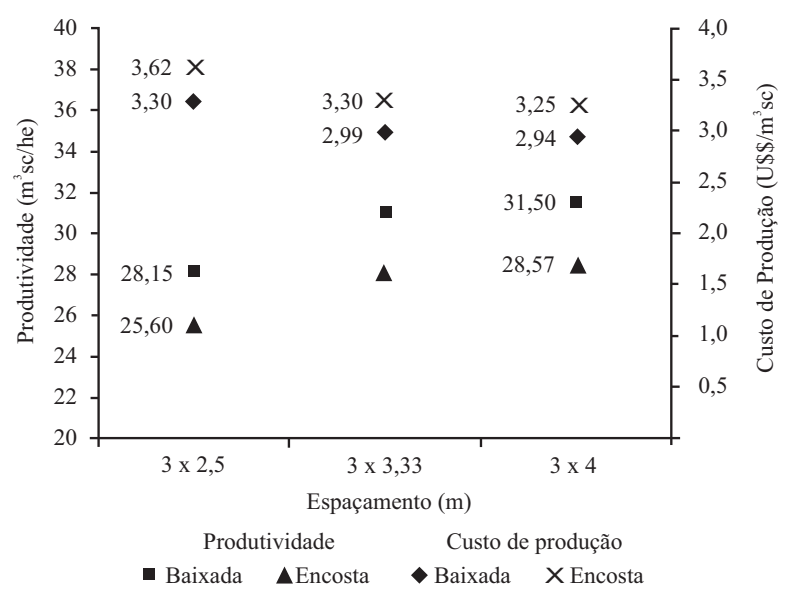

Figura 3 - Rendimento operacional e custo de produção do Harvester em função das variáveis testadas.

Figure 3-Income and production operating cost of the Harvester as a function of the variables tested.

alcançou produtividade do harvester variando entre 18,72 e $39,71 \mathrm{~m}^{3} \mathrm{sc} \mathrm{h}^{-1}$, quando as declividades oscilaram de 10 a $25 \%$ em árvores com volume médio de $0,33 \mathrm{~m}^{3}$, ou seja, a produtividade alterou-se inversamente ao percentual da declividade. Lopes et al. (2007), trabalhando com harvester na declividade de 3,5\% e volume por árvore de $0,5 \mathrm{~m}^{3}$, encontraram rendimento de $31,6 \mathrm{~m}^{3}$ $\mathrm{h}^{-1}$, confirmando os dados encontrados neste trabalho.

Esse fato é justificado pelos elementos do ciclo operacional e pelo fato de o volume por árvore ser maior e o número de árvores por hectare ser menor em espaçamentos maiores. Bramucci e Seixas (2002), testando a produtividade de diferentes harvesters, comprovaram que o volume médio das árvores é a variável que melhor explicou $80 \%$ de capacidade produtiva das máquinas. Martins et al. (2009), avaliando o harvester Timberjack 1270, encontraram sua produtividade variando com o espaçamento e volume por árvore.

Em estudos do "Forest Engineering Institute of Canada” (FERIC) sobre o desempenho de harvesters e processadores mecânicos, Richardson e Makkonen (1994) concluíram que o fator que mais afetava a capacidade produtiva de ambas as máquinas era o volume médio por árvore, e Burla (2008) completou dizendo que a declividade do terreno também é fator que influencia o rendimento operacional do harvester na colheita de florestas de eucalipto.
Em comparação com um feller-buncher, em condições de diferentes espaçamentos, Valverde et al. (1996) obtiveram melhor desempenho nas áreas com maiores espaçamentos, evidenciando que condições de espaçamento de plantio influenciam a produtividade de máquinas de corte florestal.

Silva et al. (2010), ao estudarem o harvester de esteira em condições de colheita de Pinus sp, chegaram à produtividade de $22,71 \mathrm{~m}^{3} \mathrm{he}^{-1}$, o que comprova que a produtividade da máquina aumenta com árvores de maior volume, destacando a importância de se obterem florestas de maior produtividade.

\section{CONCLUSÕES}

A atividade de traçamento consumiu maior tempo médio na colheita de árvores com o harvester. Espaçamentos menores e declividades mais acentuadas influenciam o maior consumo médio de tempo de uma mesma atividade do ciclo operacional.

As variáveis de declividade e espaçamentos influenciam significativamente a produtividade do harvester. Seu rendimento tende a ser maior com o aumento do espaçamento de plantio e a diminuição da declividade do terreno.

A mudança de espaçamento, em média, pode gerar impacto no custo de $11 \%$ e $2 \%$ a maior se a opção for pelo uso dos espaçamentos de $3 \mathrm{~m}$ x 2,5 m e $3 \mathrm{~m}$ x 3,33 $\mathrm{m}$, em comparação com o espaçamento de $3 \mathrm{~m}$ x $4 \mathrm{~m}$. Em relação à declividade, o aumento no custo gerado pela encosta é de $11 \%$ em relação à baixada. O menor custo foi no bloco de baixada e no tratamento de maior espaçamento.

\section{AGRADECIMENTOS}

À Fundação de Amparo à Pesquisa do Estado de Minas Gerais (FAPEMIG) e à Celulose Nipo-Brasileira S.A. - CENIBRA, pelo apoio e pela oportunidade de realização deste trabalho.

\section{REFERÊNCIAS}

ASSOCIAÇÃO BRASILEIRA DE PRODUTORES DE FLORESTAS PLANTADAS - ABRAFF.

Anuário estatístico da ABRAFF: anobase 2008. Brasília, 2009. 120p. Disponível em: <http://www.abraflor.org.br/estatistica.asp>. Acesso em: 19 set. 2009. 
AKAY, A.E.; ERDA, O.; SESSIONS, J. Determining productivity of mechanized harvesting machines. Journal of Applied Sciences, v.4, n.1, p.100-105, 2004.

BRAMUCCI, M.; SEIXAS, F. Determinação e quantificação de fatores de influência sobre a produtividade de "harvesters" na colheita florestal. Scientia Florestais, n.62, p.62-74, 2002.

BURla, E. R. Avaliação técnica e econômica do "harvester" na colheita do eucalipto. 2008. 62f. Dessertação (Mestrado em Engenharia Agrícola) - Universidade Federal de Viçosa, Viçosa, MG, 2008.

ELIASSON, L. Simulation of thinning with a single-grip harvester. Forest Science, v.45, n.1, p.26-34, 1999.

GINGRAS, J. F. The effect tree-length harvesting using a Keto 150 harvester heard. FERIC Field Note: Felling, n.11, p.1-2, 1992.

HUYLER, N. K.; LEDOUX, C. B. Performance of a cut-to-length harvester in a single-tree and group-selection cut. USDA Forest Service NE Research Paper, n.711, p.1-6, 1999.

JACOVINE, L. A. G. et al. Avaliação da qualidade operacional em cinco subsistemas de colheita florestal. Revista Árvore, v.29, n.3, p.391-400, 2005.

LIMA, J. S. S.; LEITE, A. M. P. Mecanização. In: MACHADO, C..C (Org). Colheita florestal. 2.ed. Viçosa, MG: Universidade Federal de Viçosa, 2008. p.43-65.

LOPES, E. S. et al. Avaliação técnica e econômica do corte de madeira de pinus com cabeçote harvester em diferentes condições operacionais. Floresta, v.37, n.3, set./dez., 2007.

MALINOVSKI, R. A. et al. Análise das variáveis de influência na produtividade das máquinas de colheita de madeira em função das características físicas do terreno, do povoamento e do planejamento operacional florestal. Floresta, v.36, n.2, p.166-182, 2006.
MARTINS, R. J.; SEIXAS, F.; STAPE, J. L. Avaliação técnica e econômica de um harvester trabalhando em diferentes condições de espaçamento e arranjo de plantio em povoamento de eucalipto. Scientia Florestais, v.37, n.83, p.253-263, 2009.

MinetTe, L. J. Avaliação técnica e econômica dos tratores florestais (forwarders), na extração de madeira de eucalipto. 1988. 77f. Dissertação (Mestrado em Ciência Florestal) - Universidade Federal de Viçosa, Viçosa, MG. 1988.

PULKKI, R. E. Glossary of forest harvesting terminology. 2006. Disponível em: <flash.lakeheadu.ca/ repulkki/

REP_terminology.pdf > Acesso em: 15 out. 2009.

RICHARDSON, R.; MAKKONEN, I. The performance of cut-to-length systems in Eastern Canada. FERIC Technical Report, n.109, p.1-16, 1994.

SANT'ANNA, C. M. Corte florestal. In: MACHADO, C. C. Colheita florestal. 2.ed.Viçosa, MG: Universidade Federal de Viçosa, 2008. p.66-69.

SILVA, E. N. et al. Avaliação técnica e econômica do corte mecanizado de Pinus sp. com harvester. Revista Árvore, v.34, n.4, p.745-753, 2010.

SILVA, M. L.; MIRANDA, G. M.; CORDEIRO, S. A. Custos. In: MACHADO, C. C (Org). Colheita florestal. 2.ed. Viçosa, MG: Universidade Federal de Viçosa, 2008. p.231-260.

SIMÕES, D.; FENNER, P. T. Influência do relevo na produtividade e custos do harvester. Scientia Forestalis, v.38, n.85, p.107-114, 2010.

STAMPFER, K.; LOSCHEK, J. Harvester operations increase productivity of cable extraction systems. Österreichische Forstzeitung (Arbeit im Wald), v. 110, n. 2, p. 4-6, 1999.

VALVERDE, S. R. et al. Análise técnicoeconômica do corte de madeira com trator florestal derrubador-amontoador (feller-buncher) no sistema de colheita florestal de árvores inteiras de eucalipto. Revista Árvore, v.20, n.2, p.229-240, 1996.

Revista Árvore, Viçosa-MG, v.38, n.1, p.000-000, 2014 
